\title{
Technical note: A simple rumen collection device for calves: An adaptation of a manual rumen drenching system ${ }^{1}$
}

\author{
R. N. Klopp, ${ }^{*}$ M. J. Oconitrillo, ${ }^{*}$ A. Sackett, ${ }^{*}$ T. M. Hill,† R. L. Schlotterbeck, $†$ and G. J. Lascano* \\ *Department of Animal and Veterinary Sciences, Clemson University, Clemson, SC 29634 \\ †Nurture Research Center, Provimi, Brookville, OH 45309
}

\begin{abstract}
A limited amount of research is available related to the rumen microbiota of calves, yet there has been a recent spike of interest in determining the diversity and development of calf rumen microbial populations. To study the microbial populations of a calf's rumen, a sample of the rumen fluid is needed. One way to take a rumen fluid sample from a calf is by fistulating the animal. This method requires surgery and can be very stressful on a young animal that is trying to adapt to a new environment and has a depressed immune system. Another method that can be used instead of fistulation surgery is a rumen pump. This method requires a tube to be inserted into the rumen through the calf's esophagus. Once inside the rumen, fluid can be pumped out and collected in a few minutes. This method is quick, inexpensive, and does not cause significant stress on the animal. This technical note presents the materials and methodology used to convert a drenching system into a rumen pump and its respective utilization in 2 experiments using dairy bull calves.
\end{abstract}

Key words: calf, rumen fluid, simple technique, rumen pump

\section{Technical Note}

Rumen fluid collection in calf nutrition research is essential to study the function of the digestive system and the rumen microbial community. The rumen microbiome is composed of bacteria, archaea, protozoa, and fungi, and these communities, by their metabolic activities, transform diet components into VFA, microbial protein, and other metabolites that will serve as a source of energy, AA, and growth promoters to the animal (Ribeiro et al., 2017). Several techniques have

Received November 27, 2017.

Accepted February 23, 2018.

${ }^{1}$ This paper is a component of USDA Regional Research Project NC-2042; Management Systems to Improve the Economic and Environmental Sustainability of Dairy Enterprises.

${ }^{2}$ Corresponding author: glascan@clemson.edu been designed to study the rumen microbial population and its changes between the different stages of the calf. Fistulas are commonly used for these types of studies, as they simplify the collection of rumen fluid, but one of their main disadvantages is the invasiveness of the surgery during early stages of development in calves (Thyfault et al., 1975). They can also be cost prohibitive compared with a rumen pump and put more stress on the animal when the calf is at the early stages of developing an active immune system.

A technique previously used in different studies for rumen fluid sample collection is the use of oral stomach tubes attached to a syringe (Abdelgadir et al., 1996). Geishauser (1993) designed an alternative technique for ruminal fluid sampling, an oro-ruminal probe with a suction pump. Nevertheless, it presents a significant limitation when collecting samples from animals with limited fluid present in the ventral sac of the rumen such as the case of young calves; the holes in the probe's head and in the suction tube tend to get obstructed. Terré et al. (2013) compared samples obtained from both ruminal fistulas and oro-ruminal tubes, finding no significant difference between the $\mathrm{pH}$ or VFA profiles; this implies that little sample integrity is sacrificed when using a stomach tube. Moreover, Paz et al. (2016) used a modified esophageal tube attached to a metal strainer (Raun and Burroughs, 1962) using suction from a vacuum pump and observed no differences in the structure of rumen bacteria communities when compared with samples taken from the same animals through rumen cannulas. Another alternative is rumenocentesis (rumen puncture), an invasive technique that requires surgical centesis of the rumen and suffers the risk of localized abscesses or peritonitis (Duffield et al., 2004) and might not be feasible in calves less than $90 \mathrm{~d}$ old.

Here, we describe a device to collect rumen samples from postweaned calves (Figure 1) created from a modification of the Cattle Pump System designed for drenching fluids into the rumen of adult cattle (Springer Magrath, Glencoe, MN). This device has been used in calves as early as $15 \mathrm{~d}$ old, but intensive 
A

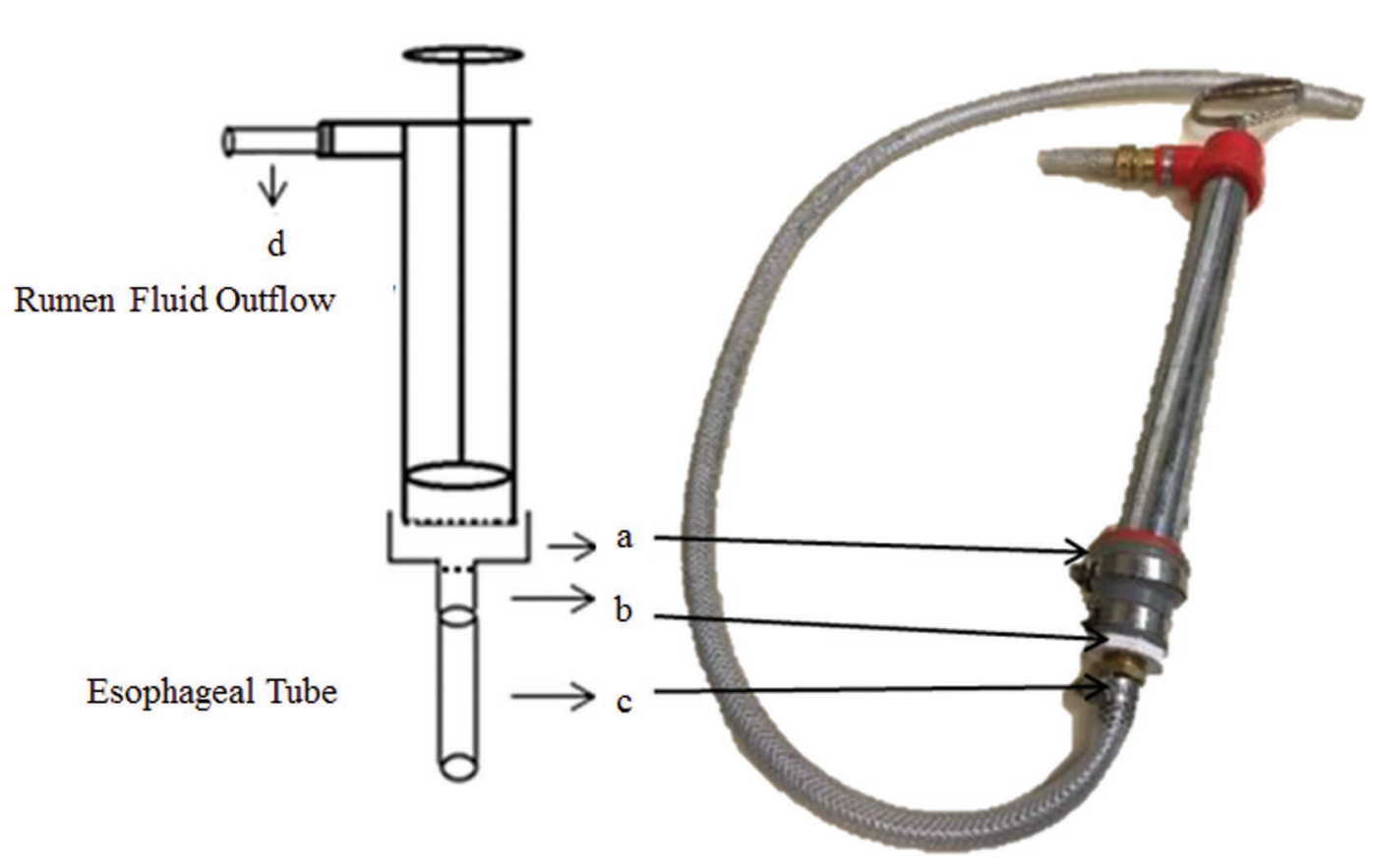

Figure 1. Detailed structure of the modified rumen collection device. (A) Diagram of the apparatus with separated parts. (B) Actual apparatus with assembled parts. (a) Flexible polyvinyl chloride (PVC) coupling $(31.75 \times 19.05 \mathrm{~mm})$ connecting end of pump to (b) PVC nonthreaded to threaded female coupling (19.05 to $15.88 \mathrm{~mm}$ ), (c) bronze male bushing threaded to a hose adapter (15.88 mm) connected to braided vinyl tubing (long hose), (d) short hose $(8 \mathrm{~cm}$ long $\times 76.2 \mathrm{~mm}$ i.d.) where sample is collected. Color version available online.

sampling has not been conducted with calves this age. The adaptations described here allow portability, sufficient vacuum for an efficient extraction, quickness $(\sim 5$ $\mathrm{min} /$ extraction), and collection of up to $250 \mathrm{~g}$ of rumen contents in calves. Although this method may be useful, for microbial identification, caution needs to be taken for fermentation profiling due to the possibility of saliva contamination.

Figure 1 depicts the assembling and modifications made to the device. The main modification was to adapt a collecting tube to the extracting end of the device. Therefore, a polyvinyl chloride flexible coupling was added to cover the drench extracting end of the pump (flexible rubber with filter) without removing the filter (inclusion is recommended if adapting a different device that does not have this filter), as it prevents clogging of the pump. A hose clamp was used to secure the connector. Then, a polyvinyl chloride reducing bushing $(31.75 \times 19.05 \mathrm{~mm}$ threaded $)$ was inserted at the other end of the connector with a hose clamp to secure it (Figure 1) and a 19.05 to $15.88 \mathrm{~mm}$ bronze male bushing was threaded to it (Figure 2A). A tube $(15.88 \mathrm{~mm}$ i.d. braided vinyl tubing) was attached to the bottom of the pump using a hose clamp, and 2 holes $(7 \mathrm{~mm}$ i.d.) were drilled through the end of the tubing to prevent clogging (Figure 2B). The length of the tube may vary depending on the size of the animal, but within animals of the same age, it does not vary significantly. For the sample collection end of the device, a $7.62-\mathrm{cm}$ piece of tubing attached to the top of the pump was used (Figure 2C).

To validate the practicality of this device and technique, this pump was used during 2016 and 2017 in 2 experiments with 50 calves in each experiment (Holstein male calves $56 \mathrm{~d}$ of age, $74 \pm 2.9 \mathrm{~kg}$ of initial BW; \pm SEM). Calves were housed in group pens (4 calves/ pen within treatment) that had $6.5 \mathrm{~m}^{2}$ of outside pen space and $1.35 \mathrm{~m}^{2}$ of inside pen space bedded with wheat straw per calf. For 56 d, calves were managed in these pens. All animals were cared for as described in the Guide for the Care and Use of Agricultural Animals in Research and Teaching (FASS, 2010) and under the approval of the institutional animal care and use committee.

Rumen sampling occurred 3 times during each experiment (70, 91, and $112 \mathrm{~d})$. During sampling, while one person restrained the calf to avoid movement and misplacement of the tube (calf's nose was held in a $90^{\circ}$ angle, avoiding applying extra force that could result in passing the tube to the trachea), another person held the calf's mouth and passed the tube over the tongue, past the epiglottis, through the upper sphincter and 
A

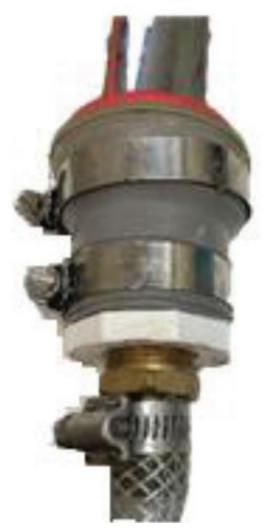

B

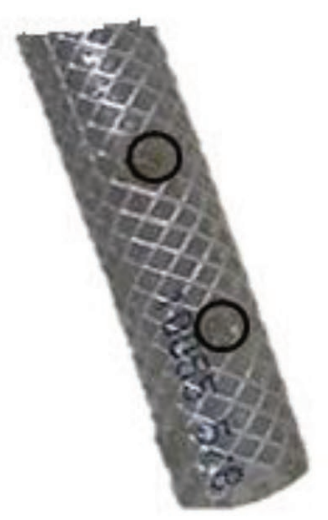

C

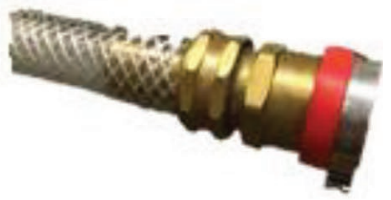

Figure 2. Position of the different parts to adapt the drenching device onto a rumen pump. (A) Flexible polyvinyl chloride (PVC) coupling (gray) attached to a PVC nonthreaded to threaded female coupling (white; $19.05 \times 15.88 \mathrm{~mm}$ ) and to a bronze male bushing threaded to a hose adapter $(15.88 \mathrm{~mm})$. (B) Holes $(7 \mathrm{~mm}$ i.d.) in collection tube that is inserted into rumen. (C) Collection end of the device; collection container to be placed under the 8-cm braided vinyl tube. Color version available online.

the cervical, thoracic, and abdominal regions of the esophagus, before reaching the rumen through the lower sphincter. At this point, slight pressure was applied to the hose to pass it down the lower sphincter into the rumen. It is helpful to measure how much tube needs to be passed down to reach the rumen and then mark it on the tube. Once the tube was far enough, and reached the site of collection, pumping was started. Pumping continued until rumen fluid was visibly flowing through the collection site of the device. Once this occurred, the pump handle was extended and held extended as the tube was being removed from the calf's esophagus. As the tube was removed, the sampling end was kept upward to avoid fluid leakage.
The first $20 \mathrm{~mL}$ of the sample was discarded to minimize saliva contamination as reported by $\mathrm{Paz}$ et al. (2016) in lactating cows but proportionally to calf's rumen volume. Approximately $40 \mathrm{~mL}$ of rumen fluid was collected (but up to $250 \mathrm{~mL}$ can be easily extracted) in a 50-mL centrifuge tube and frozen at different temperatures depending on the purpose of the future analysis. Between animals, the pump was washed thoroughly with warm water. This whole process lasted around $5 \mathrm{~min}$, did not require the use of an electrically operated vacuum pump (Paz et al., 2016), and no clogging was experienced (Geishauser, 1993). During both experiments, no problems were observed related to decreased DMI, visible indicators of stress, or calf

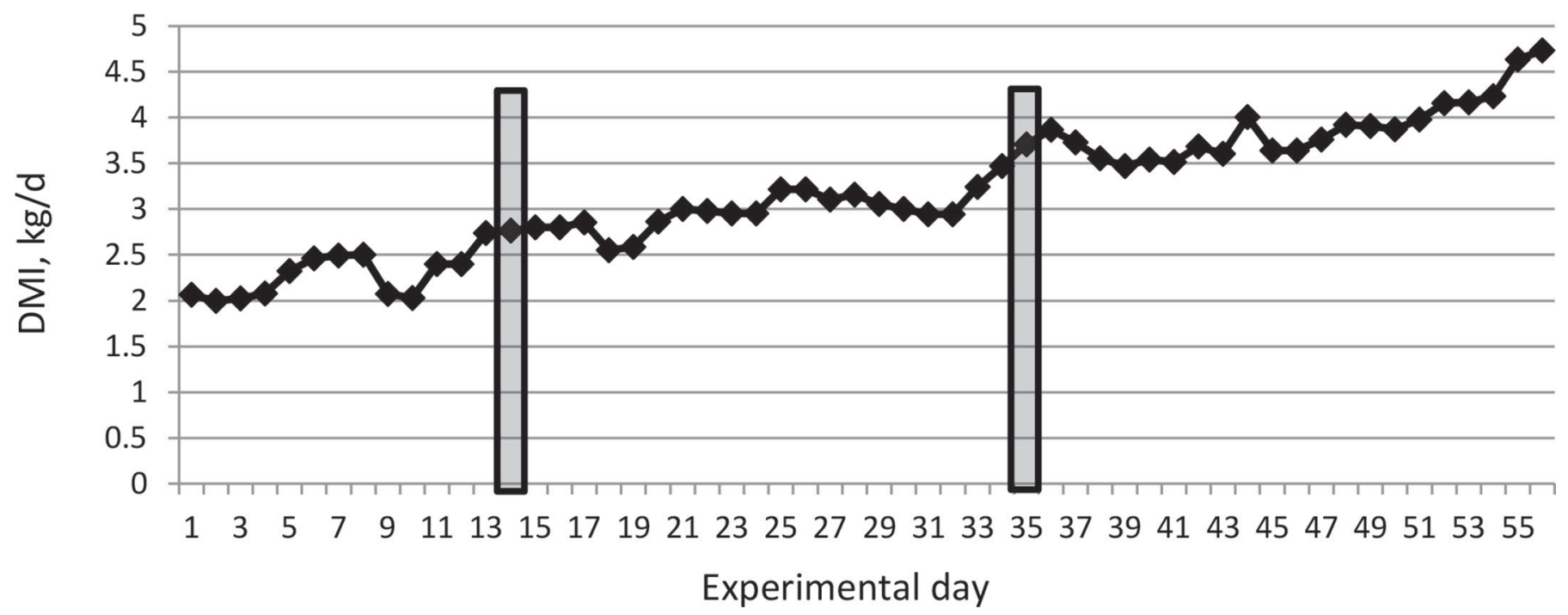

Figure 3. Dry matter intake during sampling periods on d 14 and 35 of experiment 2. Sampling times are depicted by gray bars. 
behavior. Figure 3 depicts DMI during sampling for experiment 2; DMI of the previous intake was 1.01 and $1.25 \pm 0.1 \%$ for the first sampling and 6.79 and 4.30 $\pm 0.3 \%( \pm \mathrm{SEM})$ for the second sampling for the day before and after sampling, respectively. Intake variation shown throughout the sampling time is similar to what was observed throughout the experiment.

A rumen pump adapted from a drenching device is an effective tool that allows investigators to successfully collect rumen fluid from calves. It puts limited stress on the animal and does not involve a recovery period. It is not cost prohibitive and results in quick collection intervals. This rumen pump will make it feasible for laboratories to collect and analyze rumen fluid with potential applications in investigations of rumen microbial communities, development, and establishment.

\section{ACKNOWLEDGMENTS}

Funding provided by the Clemson University Ruminant Nutrition Research Laboratory (Clemson, SC). Appreciation is extended to Clemson University Lamaster Dairy Research Center for supplying animals for initial development and Provimi Nurture Research Center personnel for sampling assistance.

\section{REFERENCES}

Abdelgadir, I. E. O., J. L. Morrill, and J. J. Higgins. 1996. Effect of roasted soybeans and corn on performance and ruminal and blood metabolites of dairy calves. J. Dairy Sci. 79:465-474.

Duffield, T., J. C. Plaizier, A. Fairfield, R. Bagg, G. Vessie, P. Dick, J. Wilson, J. Aramini, and B. McBride. 2004. Comparison of techniques for measurement of rumen $\mathrm{pH}$ in lactating dairy cows. J. Dairy Sci. 87:59-66.

FASS. 2010. Guide for the care and use of agricultural animals in research and teaching. 3rd ed. FASS Inc., Champaign, IL.

Geishauser, T. 1993. An instrument for collection and transfer of ruminal fluid and for administration of water soluble drugs in adult cattle. Bov. Pract. 27:38-42.

Paz, H. A., C. L. Anderson, M. J. Muller, P. J. Kononoff, and S. C. Fernando. 2016. Rumen bacterial community composition in Holstein and Jersey cows is different under same dietary condition and is not affected by sampling method. Front. Microbiol. 7:1206.

Raun, N. S., and W. Burroughs. 1962. Suction strainer technique in obtaining rumen fluid samples from intact lambs. J. Anim. Sci. $21: 454-457$

Ribeiro, G. O.. D. B. Oss, Z. He, R. J. Gruninger, C. Elekwachi, R. J. Forster, W. Yang, K. A. Beauchemin, and T. A. McAllister. 2017. Repeated inoculation of cattle rumen with bison rumen contents alters the rumen microbiome and improves nitrogen digestibility in cattle. Sci. Rep. 7:1276-1292.

Terré, M., L. Castells, F. Fàbregas, and A. Bach. 2013. Short communication: Comparison of $\mathrm{pH}$, volatile fatty acids, and microbiome of rumen samples from preweaned calves obtained via cannula or stomach tube. J. Dairy Sci. 96:5290-5294.

Thyfault, H. A., E. C. Leffel, and M. G. Huang. 1975. Simplified method for producing permanent ruminal fistulae. J. Dairy Sci. $58: 1899-1901$ 